\title{
Predicting the Actual Strength of Open-End Spun Yarn Using Mechanical Model
}

\author{
Resty Mayseptheny Hernawati ${ }^{1, a}$,Valentinus Galih Vidia Putra ${ }^{2, b}$ \\ and Irfandhani Fauzi ${ }^{3, c}$ \\ 1, 2, 3Sekolah Tinggi Teknologi Tekstil, Bandung, Indonesia \\ aresty.mayseptheny.h@gmail.com, bgalih_vidia@yahoo.com, ‘fauzi.fandhani@gmail.com
}

\begin{abstract}
Keywords:predicting, yarn, strength, rotor speed, rotor diameter, rotor, take-off nozzle, Open-End spun yarn
\end{abstract}

\begin{abstract}
On this journal, modeling relation of rotor speed and rotor diameter toward yarn tension on take-off nozzle has been validated by theoretical approach and numeric computation.It shows that value of yarn tension is $\mathrm{F}=\left(1,4.10^{-13}\right) \mathrm{Tt}_{\mathrm{R}}{ }^{2} \mathrm{~d}_{\mathrm{R}}^{2}$ [cN]. Excessively high spinning tension always results in an intolerable increase in yarn breakage. This paper presents a comparative study of theoretical and reference-based experimental result for predicting the actual strength of open-end spinning system.
\end{abstract}

\section{Introduction}

According to Lawrence[1], Open-End (OE) spinning has several advantages over ring spinning, such as increased production rate, separation of twisting and winding, possibilities of full automation of yarn spinning, and elimination of speed frame and winding. Rotor spinning, friction spinning and vortex spinning systems are three major methods of yarn manufacture developed on the principle of open end spinning. According to Gunter Trommer[3], the characteristic of all OE spinning processes is the teasing out of the sliver into individual fibres, followed by reassembly of the fibres and their twisting into the end of the yarn, enabling the twist imparting and yarn winding processes to be performed independently by two different mechanisms. The essential features of the open end spinning process are thus drafting, fibre transport, fibre alignment, cleaning (if necessary), fibre condensation, twisting, yarn removal and winding[1]. Individual fibres are being twisted at the yarn peel-off point. According to Vaclav Rohlena[2] and Gunter Trommer[3], tenacity [cN] has tendency to arise until take-off nozzle point. According to Gunter Trommer[3], value of yarn tension is $\left(1,4.10^{-13}\right) \mathrm{Tt} \mathrm{n}_{\mathrm{R}}^{2} \mathrm{~d}_{\mathrm{R}}^{2}[\mathrm{cN}]$.

Spinning tension, which inevitably increases as rotor speed increases with constant rotor diameter in the rotor spinning process, is ultimately limited by fibre strength. Excessively high spinning tension always results in an intolerable increase in yarn breakage. Tension yarn breaks take place in the already spun yarn, normally between the take-off nozzle and take-up rollers, leaving a short, broken yarn end in the rotor groove. The cause of tension yarn breaks is always excessive spinning tension, which affects the weakest yarn point i.e. the point, between the take-off nozzle and the take-up roller. According to Gunter Trommer[3], yarn tension in the centre of the take-off nozzle should therefore no exceed from 1/10 until 1/15 of the fibre strength.

In addition to yarn quality, running performance plays a major role in evaluation of a spinning process. Running behavior is often expressed in terms of the number of yarn breaks per unit mass of yarn. According to Lawrence[1], the stability of the rotor spinning process decides whether a problem such as trash particles, foreign fibres, dust,etc., will result in a yarn break or not. Spinning stability in rotor spinning is largely influenced by the following four factors. One of those factors is $\Sigma$ the numbers of fibres in the yarn cross-section. According to Gunter Trommer[3], finer fibres permit a correspondingly finer yarn count in accordance with the minimum number of around $\mathrm{Z}>$ 900-110 fibres necessary on average in the yarn cross-section in rotor spinning. 
Experimental result by Erdem Koç, Carl Anthony Lawrence, and Cherian Iype published by FIBRES \& TEXTILES in Eastern Europa[4] .Their yarn samples were produced under different running conditions using different rotor diameters $(32 \mathrm{~mm}$ to $56 \mathrm{~mm}$ ) with different rotor speed ranging from $52,200 \mathrm{rpm}$ to $69,920 \mathrm{rpm}$ on a Rieter R1 OE-Rotor Spinning Unit. Their yarn samples were $100 \%$ PES and had approximately 25 Tex linear density. The summary of their experimental results are showed at Table 1 and Table 2.

Table 1. Sample yarns produced on OE-rotor unit (R1)[4]

\begin{tabular}{|c|c|c|c|c|}
\hline \multirow{2}{*}{$\begin{array}{c}\text { Rotor speed } \\
{[\mathrm{rpm}]}\end{array}$} & \multicolumn{4}{|c|}{ Rotor diameters [mm] } \\
\cline { 2 - 5 } & A (32) & B (40) & C (48) & D (56) \\
\hline I, 52 200 & I A & I B & I C & I D \\
\hline II, 60070 & II A & II B & II C & II D \\
\hline III, 69920 & III A & III B & III C & III D \\
\hline
\end{tabular}

Table 2. Strength test result of yarn samples [4]

\begin{tabular}{|c|c|c|c|c|}
\hline \multirow{2}{*}{$\begin{array}{c}\text { Yarn } \\
\text { sample }\end{array}$} & \multicolumn{2}{|c|}{ Yarn Force } & \multicolumn{2}{c|}{ Yarn Tenacity } \\
\cline { 2 - 5 }$[\mathrm{cN}]$ & $\mathrm{CV}_{\mathrm{F}}[\%]$ & {$[\mathrm{cN} / \mathrm{Tex}]$} & $\mathrm{CV}[\%]$ \\
\hline I A & 459.74 & 6.91 & 19.64 & 6.91 \\
\hline I B & 499.59 & 5.84 & 21.23 & 5.84 \\
\hline I C & 490.58 & 7.80 & 21.06 & 7.80 \\
\hline I D & 454.77 & 6.79 & 19.52 & 6.79 \\
\hline II A & 489.97 & 6.66 & 21.06 & 6.66 \\
\hline II B & 516.99 & 7.78 & 22.51 & 7.78 \\
\hline II C & 508.10 & 7.06 & 22.02 & 7.06 \\
\hline II D & 402.77 & 11.69 & 17.14 & 11.69 \\
\hline III A & 516.79 & 9.63 & 22.09 & 9.63 \\
\hline III B & 523.40 & 8.09 & 22.26 & 8.09 \\
\hline III C & 456.98 & 9.14 & 19.60 & 9.14 \\
\hline
\end{tabular}

Table 1 explains conditions which is the yarn samples were produced using different rotor diameter and different rotor speed. As can be seen in Table 2, yarn tension and yarn tenacity increase as rotor speed increases with constant rotor diameter in the rotor spinning process, i.e. for rotor diameter $32 \mathrm{~mm}$ (A), the yarn tension for IA; IIA; and IIIA are $456.74 \mathrm{cN}, 19.64 \mathrm{cN} / \mathrm{Tex}$; $489.97 \mathrm{cN}, 21,6 \mathrm{cN} / \mathrm{Tex}$; and $516.79 \mathrm{cN}, 22.09 \mathrm{cN} / \mathrm{Tex}$.

\section{Modeling of Yarn Movement on Rotor}

On Z-Twisted type can be analyzed as follow:

Assuming that angular speed of rotor movement is $\phi^{\prime}$ and speed of yarn movement is $\psi^{\prime}$, these movement move at same direction as showed at Figure1.
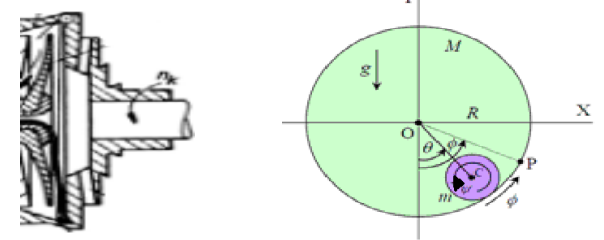

Fig. 1 Yarn and rotor movement schema

Thus it can be explained that yarn and rotor are assuming as a rigid cylinder with mass $\mathrm{m}$ and $\mathrm{M}$, radius of yarn is $r$, and radius of rotor is $R$. 


$$
\begin{aligned}
& (R-r) \theta^{\prime}=\psi^{\prime} r+R \phi^{\prime}=V d+R \phi^{\prime} \\
& V d=(R-r) \theta^{\prime}-R \phi^{\prime}
\end{aligned}
$$

Assuming that yarn radius (r) is much smaller than rotor radius (R), then equation (2) can be written as

$$
\begin{aligned}
& V d=\left(\theta^{\prime}-\phi^{\prime}\right) R \\
& V d=\left(n_{\text {yarn }}-n_{\text {rotor }}\right) \frac{\lambda \pi}{180^{0}} R \\
& V d=\frac{\left(n_{\text {yarn }}-n_{\text {rotor }}\right)}{360^{0}} \lambda \pi d
\end{aligned}
$$

$\mathrm{Vd}=$ yarn speed $(\mathrm{m} / \mathrm{s})$

$\mathrm{n}_{\text {yarn }}, \mathrm{n}_{\text {rotor }}=$ yarn and rotor rotation per time $(1 / \mathrm{s})$

$\mathrm{R}=$ radius of rotor $(\mathrm{m})$

$\mathrm{d}=$ rotor diameter $(\mathrm{m})$

If angle of (5) is $360^{\circ}$, it can be written as

$$
V d=\left(n_{\text {yarn }}-n_{\text {rotor }}\right) \pi d
$$

\section{Modeling Yarn Tension on Rotor}

Study analysis of yarn movement mechanic on rotor is explained on Figure 2 below. The purpose is to get a movement equation of yarn tension.

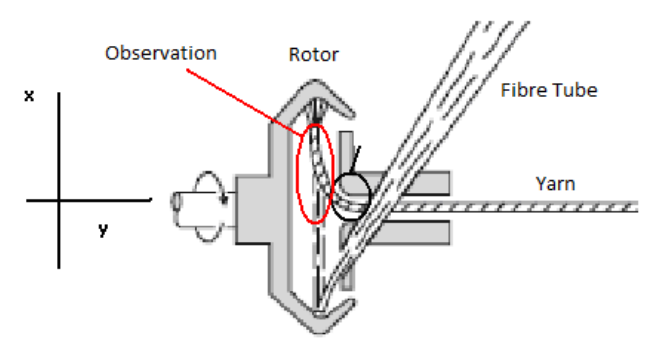

Fig. 2 Yarn tension on rotor schema

Observation: Assuming that there is rotation of Oxyz framework (rotor) which is affect yarn at $\phi^{\prime}$, while $\mathrm{x}$-axis is along with yarn movement, and it has rotation on y-axis. It causes centrifugal force which is will equalize tension force on yarn. According to Gunter Trommer[3], yarn tension increases as rotor speed increases with constant rotor diameter in the rotor spinning process.

$$
\begin{aligned}
& \sum F=M R \phi^{\prime 2} \\
& \Delta T_{R}=\Delta M R \phi^{\prime 2}=\varsigma . \Delta R R \phi^{\prime 2} \\
& \Delta T=\varsigma . \Delta x \cdot x \phi^{\prime 2} \\
& \frac{\Delta T}{\Delta x}=\varsigma \cdot x \phi^{\prime 2} \\
& \frac{\partial T}{\partial x}=\varsigma \cdot x \phi^{\prime 2}
\end{aligned}
$$




$$
\begin{aligned}
& d T=\int_{x=\rho}^{R} \varsigma \cdot x \phi^{\prime 2} d x \\
& \Delta T=T_{\rho}-T_{o}=\frac{\varsigma}{2}\left(R^{2}-\rho^{2}\right) \phi^{\prime 2} \\
& \frac{T_{\rho}}{\varsigma}-\frac{T_{o}}{\varsigma}=\frac{1}{2}\left(R^{2}-\rho^{2}\right) \phi^{\prime 2} \\
& \frac{T_{\rho}}{\varsigma} \approx\left(\frac{1}{2}\right)\left(R^{2}-\rho^{2}\right) \phi^{\prime 2} \\
& \frac{T_{\rho}}{\varsigma}=\operatorname{Const}\left(R^{2}-\rho^{2}\right) \\
& \text { For } ; \rho \approx 0 \\
& \frac{T_{\rho}}{\varsigma}=\frac{T_{\rho}}{T_{t 2}} \approx \frac{1}{2} R^{2} \phi^{\prime 2} \\
& T_{\rho} \approx \frac{1}{2} T_{t 2} R^{2} \phi^{\prime 2}
\end{aligned}
$$

If angular speed of rotor for one circle is $\phi^{\prime} \frac{\lambda \pi}{360}=\frac{360}{360} \pi$ per minute (60 second) and unit of rotor diameter is $\mathrm{mm}\left(10^{-3}\right.$ meter), then the Constanta for strength is by converting units to SI units as:

$$
\begin{aligned}
& T_{\rho} \approx \frac{1}{2} T_{t 2} R^{2} \phi^{\prime 2}\left(\pi \frac{1}{60} 10^{-3}\right)^{2} \\
& T_{\rho} \approx \frac{1}{2} T_{t 2} R^{2} \phi^{\prime 2}\left(2,7.10^{-15}\right) \\
& T_{\rho} \approx 1,4.10^{-15} T_{t 2} R^{2} \phi^{\prime 2}[N]
\end{aligned}
$$

If we convert $[\mathrm{N}]$ to $[\mathrm{cN}]$, then the Constanta for strength is

$$
T_{\rho} \approx 1,4 \cdot 10^{-13} T_{t 2} R^{2} \phi^{\prime 2}[c N]
$$

\section{Result and Discussion}

\subsection{Predicting Yarn Strength}

According to Gunter Trommer[3], yarn tension in the centre of the take-off nozzle should therefore no exceed from $1 / 10$ untill/15 of the fibre strength $\left(\mathrm{F}_{\mathrm{F}}\right)$. This expression can be written as maximum permissible spinning tension.

$F \max =\frac{1}{U} F_{F}$

According to Gunter Trommer[3], Fmax $=\left(1,4.10^{-13}\right) \mathrm{n}_{\mathrm{R}}{ }^{2} \mathrm{~d}_{\mathrm{R}}{ }^{2}$ [cN], then

$$
\left(1.4 .10^{-13}\right) \mathrm{n}_{\mathrm{R}}^{2} \mathrm{~d}_{\mathrm{R}}^{2}[\mathrm{cN}] \leq \frac{1}{U} F_{F}
$$


Assuming that samples were produced under same conditions as test result experiment by Erdem Koç et al. [4].In this predicting, value of Constanta $U$ were 10, minimum number of average in the yarn cross-section in rotor spinning $(Z) 110$ fibres. Then the strength test result (predicting result) is

$$
F_{F}=F \max \times Z
$$

Strength test result (predicting approach) can be achieved as Table 3 below.

Table 3.Strength test result (predicting result)

\begin{tabular}{|c|c|c|c|c|}
\hline \multicolumn{2}{|c|}{ Rotor } & \multicolumn{3}{c|}{ Predicting Result } \\
\hline Diameter [mm] & Speed [rpm] & Fmax & $\mathrm{F}_{\mathrm{F}}$ & Force yarn [cN] \\
\hline 32 & 52200 & 0,390633 & 3,906331 & 429,6964 \\
\hline 32 & 60070 & 0,517301 & 5,173009 & 569,031 \\
\hline 32 & 69920 & 0,700859 & 7,008593 & 770,9452 \\
\hline
\end{tabular}

Table 4 below explains comparison of yarn strength value between observation and prediction. And Figure 3 shows predicted versus observed experimental yarn strength value.

Table 4. Comparison of yarn strength

\begin{tabular}{|c|c|c|c|}
\hline \multicolumn{2}{|c|}{ Rotor } & \multicolumn{2}{c|}{ Yarn strength [cN] } \\
\hline Diameter [mm] & Speed [rpm] & Predicting Result & Experimental Result \\
\hline 32 & 52200 & 429,6964 & 459.74 \\
\hline 32 & 60070 & 569,031 & 489.97 \\
\hline 32 & 69920 & 770,9452 & 516.79 \\
\hline
\end{tabular}

As can be seen from Table 4 and Figure 3, the value of prediction and observed (actual) yarn strength has $\mathrm{R}^{2}$ value 0,980 . Therefore the prediction value is acceptable.

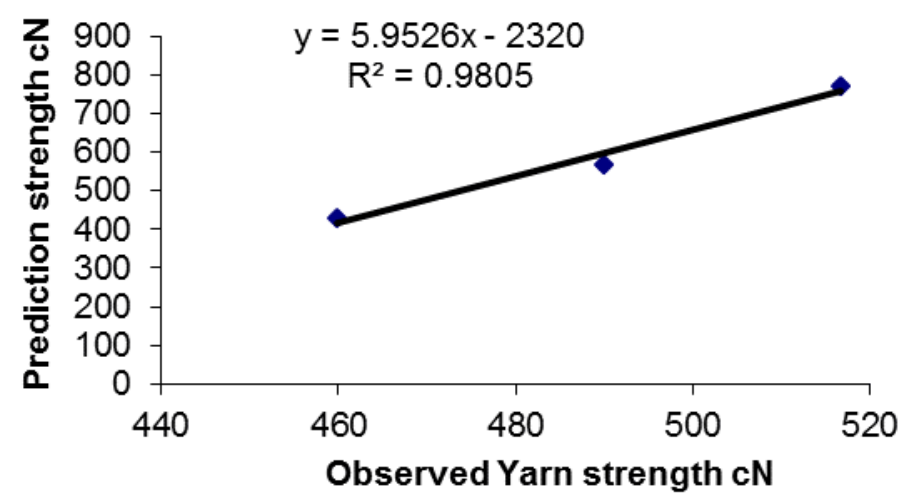

Fig. 3 Predicted versus observed yarn strength value

\subsection{Predicting Yarn Tenacity}

Assuming that samples were produced under same conditions as test result experiment by Erdem Koç et al. [4]. In this predicting, value of Constanta U were 10, minimum number of average in the yarn cross-section in rotor spinning $(Z)=110$ fibres.

According to Gunter Trommer[3], $\mathrm{Fmax}=\left(1,4.10^{-13}\right) \mathrm{Tt} \mathrm{n}_{\mathrm{R}}{ }^{2} \mathrm{~d}_{\mathrm{R}}{ }^{2}[\mathrm{cN}]$, then $\mathrm{Tt}$ as yarn count system for Tex which is yarn samples were 100\% PES and had approximately 25 tex linear density, then to achieve yarn tenacity value:

$$
T_{t}=\frac{F \max }{T e x}
$$


Table 5.Tenacity test result (predicting result)

\begin{tabular}{|r|r|r|r|}
\hline \multicolumn{2}{|c|}{ Rotor } & \multicolumn{2}{|l|}{ Predicting Result } \\
\hline Diameter [mm] & Speed [rpm] & Fmax [cN] & Tenacity [cN/Tex] \\
\hline 32 & 52200 & 0,390633 & 17.18785475 \\
\hline 32 & 60070 & 0,517301 & 22.76124076 \\
\hline 32 & 69920 & 0,700859 & 30.83780856 \\
\hline
\end{tabular}

Table 6 below explains comparison of yarn strength value between observation and prediction. And Figure 4 shows predicted versus observed experimental yarn tenacity value.

Table 6. Comparison of yarn tenacity

\begin{tabular}{|c|c|c|c|}
\hline \multicolumn{2}{|c|}{ Rotor } & \multicolumn{2}{c|}{ Yarn Tenacity [cN/Tex] } \\
\hline Diameter [mm] & Speed [rpm] & Predicting Result & Experimental Result \\
\hline 32 & 52200 & 17.18785475 & 19.64 \\
\hline 32 & 60070 & 22.76124076 & 21.06 \\
\hline 32 & 69920 & 30.83780856 & 22.09 \\
\hline
\end{tabular}

As can be seen from Table 6 and Figure 4, the value of prediction and observed (actual) yarn tenacity has $\mathrm{R}^{2}$ value 0,961 . Therefore the prediction value is acceptable.

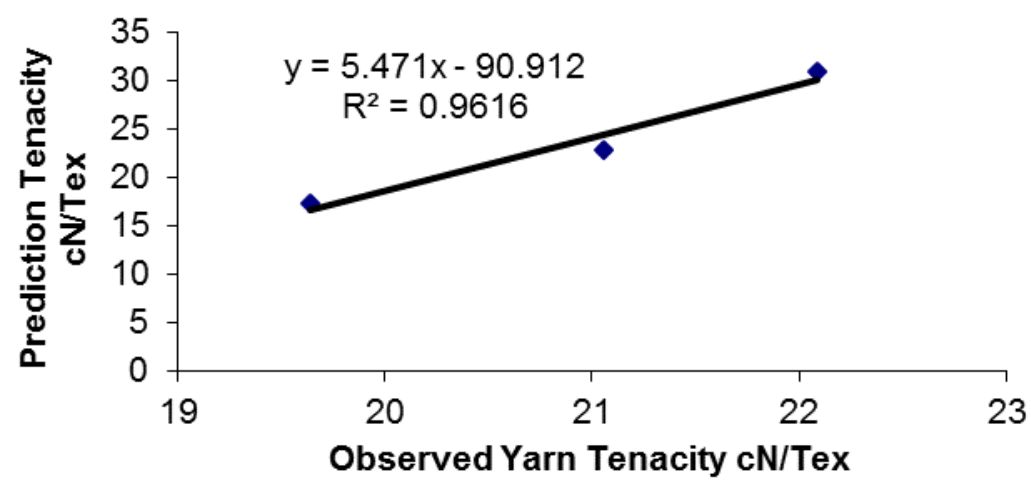

Fig. 4 Predicted versus observed yarn tenacity value

\section{Conclusions}

This modeling relation of rotor speed and rotor diameter toward yarn tension can be used to predicting yarn strength. This modeling results that yarn tension is as same as According to Gunter Trommer[3], $\mathrm{F}=\left(1,4.10^{-13}\right) \mathrm{Tt} \mathrm{n}_{\mathrm{R}}^{2} \mathrm{~d}_{\mathrm{R}}^{2}[\mathrm{cN}]$.

$\mathrm{R}^{2}$ of both yarn strength and yarn tenacity is closer to 1 , therefore this prediction is acceptable and can be used to predicting yarn strength.

\section{References}

[1] Lawrence, Advances in yarn spinning technology, The textile Institute, Cambridge, UK, 2010.

[2] Rohlena, Vaclav., Open-End Spinning, Elseiver Scientific Publishing Company, New york, 1975.

[3] Trommer, Gunter., Rotor Spinning, Deutscher fachverlag, Frankfurt, 1995.

[4] Koç, Erdem.et al.,Wrapper Fibres in Open-End Rotor-Spun Yarns: Yarn Properties and Wrapper Fibres,FIBRES \& TEXTILES in Eastern Europa,2005. 